\title{
Investigating the TLR9 $m R N A$ Expression Level in Different Histological Types of Colorectal Polyps
}

\author{
Sama Rezasoltani' ${ }^{1}$, Shirin Khatibi ${ }^{1}$, Zahra Pezeshkiyan' ${ }^{1}$, Ehsan Nazemalhosseini- \\ Mojarad $^{2 *}$, Maryam Sharafkhah ${ }^{3}$, Amir Sadeghi ${ }^{1}$, Hamid Asadzadeh Aghdaei ${ }^{1}$, \\ Mohammad Reza Zali ${ }^{2}$
}

\begin{abstract}
Toll-like receptor 9 (TLR9) is a cellular DNA receptor of the innate immune system which plays a pivotal role in inflammatory response. Recently, changing expression levels of TLR 9 has been observed in a wide range of cancer cells; however, there is little information about colorectal polyps. Herein, we assessed the mRNA expression of TLR9 in different colorectal polyp types compared to normal group in order to investigate its expression level during CRC initiation. Fifty-four biopsy samples from colorectal polyp patients and from 20 healthy subjects were collected. The mucosal mRNA expression level of TLR9 gene was identified by real time PCR. Fold change of gene expression was evaluated by $2^{-\Delta \Delta \mathrm{ct}}$ method. There was a significant relationship between the lower expression of TLR 9 gene in the polyp cases compared to normal individuals ( $\mathrm{P}$ value $=0.0005)$, Also, decreased TLR9 $m R N A$ expression was obtained in adenomas in contrast to hyperplastic and normal groups $(\mathrm{P}$ value $=0.0008)$. Based on the current results, we hypothesized that aberrant surface expression of TLR 9 on tumor cells may promote the growth and invasion of colorectal polyps. Further, TLR 9 modulation may have an important impact on the development of novel therapeutic strategies.
\end{abstract}

Keywords: Colorectal Polyp- mRNA expression- TLR9

Asian Pac J Cancer Prev, 20 (8), 2299-2302

\section{Introduction}

Colorectal cancer (CRC) is a considerable global health problem in the world and the third diagnosed malignancy following lung and breast cancer (Peters et al., 2016; Hale et al., 2017). CRC is one of the major causes of cancer death in western countries and also the third most common cancer in Iran (Ansari et al., 2006; Haghdoost et al., 2011). Most CRC cases progress from precursors recognized as colorectal polyps; the two common types of polyps include: hyperplastic (HP) and adenomatous polyps (AP). AP itself is divided in three main sub-groups of tubular adenoma (TP), tubuvillous (TVP), and villus (VP). Previous investigations (Leslie et al., 2002; Kim et al., 2011) have shown that colorectal tumors may originate from transformed polyps within several years. Histologically, colorectal polyps are found in different shapes including adenoma, hyperplastic, hamartomatous, and inflammatory polyps (Leslie et al., 2002). Neoplastic or adenoma polyps are more important as they are suspected of being malignant and cancerous (O'Connell and Crockett, 2017). Indeed, recent investigations have revealed that hyperplastic polyps have the malignancy potential in some cases (Liljegren et al., 2003; Inoue et al., 2007). The relationship between inflammation and tumor is becoming increasingly important in the study on the pathogenesis of CRC. Several studies have confirmed that TLRs as immune molecules could mediate inflammatory response and play an important role in this process (Chen et al., 2007; Kolumam et al., 2005; Siegel et al., 2016). Nevertheless, the role of TLR9-mediated immune inflammation reaction in the process is not currently clear (Caixia et al., 2018). In this study, we dealt with evaluating the expression level of the TLR9 in colonic AP and HP cases in contrast to healthy subjects and with finding the relationship between TLR9 $m R N A$ expression level and pathological features of AP and HP cases.

\section{Materials and Methods}

\section{Human sample collection}

The present study was case control and the investigated population (54 patients with polyp and 20 normal individuals) was chosen from the cases with colorectal polyps who were referred to Taleghani hospital, Shahid Beheshti University of Medical Sciences, Tehran-Iran,

${ }^{1}$ Basic and Molecular Epidemiology of Gastrointestinal Disorders Research Center, ${ }^{2}$ Gastroenterology and Liver Diseases Research Center, Research Institute for Gastroenterology and Liver Diseases, Shahid Beheshti University of Medical Sciences, ${ }^{3}$ Liver and Pancreatobiliary Diseases Research Center, Digestive Diseases Research Institute, Tehran University of Medical Sciences, Tehran, Iran.*For Correspondence: ehsanmojarad@gmail.com 
between October 2015 and April 2017. The sample was chosen through ransom sampling. Colorectal polyps were identified through colonoscopy and confirmed by pathology results. Polyp-free controls were defined as those with no polyps identified during colonoscopy and no previous history of colorectal polyps. Polyp type classifications were done according to polyp histology; hyperplasic (HP), tubular adenoma (TA), and tubulovillous polyp (TVP), where dysplasia grades and polyp sizes were characterized by a pathologist. The clinical information of patients was collected by a questionnaire. The study was approved by the Clinical Research Ethics Committee of Shahid Beheshti University of Medical Sciences and the Ethics Committee of Taleghani Hospital, Tehran, Iran with No.2014/770.

\section{RNA extraction and quality control}

Total RNA was extracted from all samples (Yekta Tajhiz Azma kit, Teheran, Iran) according to the kit instructions. RNA concentration and purity ratios (OD260/280, OD260/230) were evaluated by NanoDrop 1000 spectrophotometer (NanoDrop Technologies, Wilmington, DE, USA). The integrity of RNA was determined by electrophoresis on a denaturing $1.5 \%$ agarose gel.

\section{cDNA synthesis}

Total RNAs were converted to cDNA by Retrotransciptase (RT) reaction (TaKaRa kit, Cat No.RR037A, Otsu, Shiga, Japan) according to the following: $2 \mathrm{mg}$ of total RNA was picked up and denatured at $95^{\circ} \mathrm{C}$ for $5 \mathrm{~min}$. Thereafter, the tubes were placed on ice where $5 \mu \mathrm{L}$ of $5 \times$ primer script buffer, $0.5 \mu \mathrm{L}$ RT enzyme, $1.24 \mu \mathrm{M}$ oligo dt primer, $10 \mu \mathrm{M}$ random 6 mer, $1 \mu \mathrm{M}$ ribolock, $1 \mu \mathrm{L}$ easy dilution, and $5 \mu \mathrm{L}$ RNA free distilled water $\left(\mathrm{dH}_{2} \mathrm{O}\right)$, were added. The cDNA synthesis was performed as follows: $25^{\circ} \mathrm{C}$ for $5 \mathrm{~min}, 42^{\circ} \mathrm{C}$ for $15 \mathrm{~min}$, $85^{\circ} \mathrm{C}$ for $1 \mathrm{~min}$ for inactivation of the reverse transcriptase enzyme and $4^{\circ} \mathrm{C}$ for $10 \mathrm{~min}$ for hold temperature. Next, cDNA products were stored at $-20^{\circ} \mathrm{C}$. Note that in all reactions, the same concentrations of RNA samples were used (RNA adjustment).

\section{Real time- $P C R$}

Real-time PCR was performed following the standard SYBR Premix Ex Taq ${ }^{\mathrm{TM}}$ kit (TaKaRa Bio Inc., Otsu, Japan) protocol, using a final volume of $20 \mu \mathrm{L}$ containing $5 \mu \mathrm{L}$ of reverse-transcribed cDNA and $1 \mu \mathrm{L}$ of $10 \mathrm{pmol}$ forward and reverse primers, using Applied Biosystems 7500 version 1 (ABI, Foster City, CA, USA). Real-time PCR was carried out by expressive primers (Table 1)

Table 1. Oligonucleotides Pair of Primers Used for the Relative Gene Expression

\begin{tabular}{ll}
\hline Gene & \multicolumn{1}{c}{ Primer Sequence } \\
\hline TLR9-F & 5'_AATCCCTCATATCCCTGTCCC_3' \\
TLR9-R & 5',GTTGCCGTCCATGAATAGGAAG_3' \\
GAPDH-F & 5'-TGACTTCAACAGCGACACCCA-3' \\
GAPDH-R & 5'-CACCCTGTTGCTGTAGCCAAA-3' \\
\hline
\end{tabular}

under the following conditions: $95^{\circ} \mathrm{C}$ for $5 \mathrm{~s}, 40$ cycles of $95^{\circ} \mathrm{C}$ for $5 \mathrm{~s}, 60^{\circ} \mathrm{C}$ for $34 \mathrm{~s}, 95^{\circ} \mathrm{C}$ for $15 \mathrm{~s}, 60^{\circ} \mathrm{C}$ for 1 $\mathrm{s}$ and $60^{\circ} \mathrm{C}$ for $15 \mathrm{~s}$ (Khatibi et al., 2018). Amplification signals for samples were normalized by glyceraldehyde 3 -phosphate dehydrogenase (GAPDH) gene. Fold change of gene expression was evaluated by $2^{-\Delta \Delta c t}$ method.

Statistical analysis

All results were analyzed by Graph pad Prism software version 5 (San Diego, CA, USA). The data were non-normally distributed and the non-parametric test was used. Specifically, student t-test and one-way ANOVA test were performed. P-value $<0.05$ was considered statistically significant.

\section{Results}

Among 54 polyp cases, $26(48.3 \%)$ were identified as hyperplastic, $7(12.9 \%)$ as tubular, and $21(38.8 \%)$ as tubulovillous. Clinicopathological parameters of patients with different polyp types are presented in Table 2. Based on these results, there was a significant relationship between the lower expression level of TLR 9 in the polyp cases compared with normal samples $(P$-value $=0.0005)$ (Figure 1). Also, comparing AP (TP, TVP), HP, and

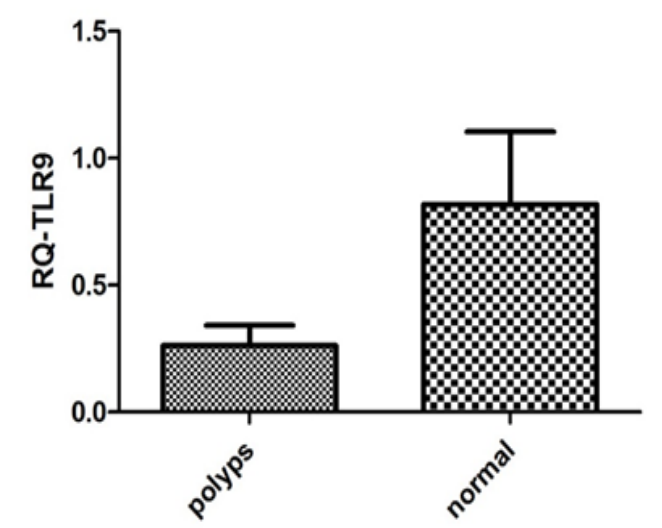

Figure 1. The Significant Down-Regulation of TLR9 mRNA in the Polyp Group Compared with the Normal Group $(P$-value $=0.0005)$

Table 2. Clinicopathological Parameters of Patient with Different Polyps

\begin{tabular}{llc}
\hline Parameter & \multicolumn{1}{c}{ Type } & Number \\
\hline Polyp types & Hyperplastic & $26(48.3 \%)$ \\
& Tubular & $7(12.9 \%)$ \\
& Tubullovillous & $21(38.8 \%)$ \\
Dysplasia & HGD & $24(44.5 \%)$ \\
& LGD & $30(55 . \%)$ \\
Gender & Male & $28(52 \%)$ \\
& Female & $26(48 \%)$ \\
Site & Rectum & $16(29.6 \%)$ \\
& AC & $7(13 \%)$ \\
& AD & $15(27.8 \%)$ \\
Total & TC & $16(29.6 \%)$ \\
\hline
\end{tabular}




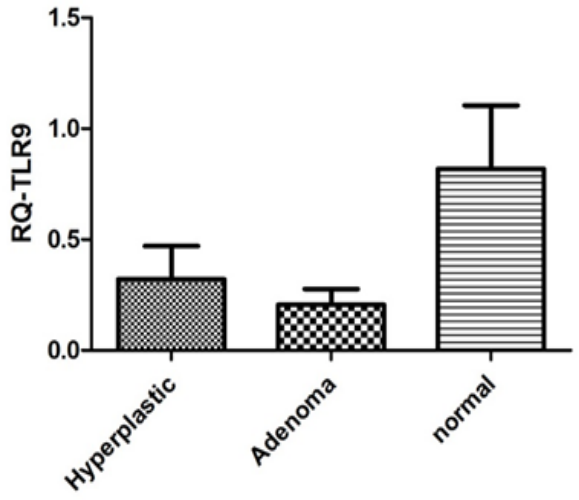

Figure 2. The Significant Down-Regulation of TLR9 mRNA in Adenoma Polyps Compared with the to Hyperplastic and Normal Groups $(P$-value $=0.0008)$.

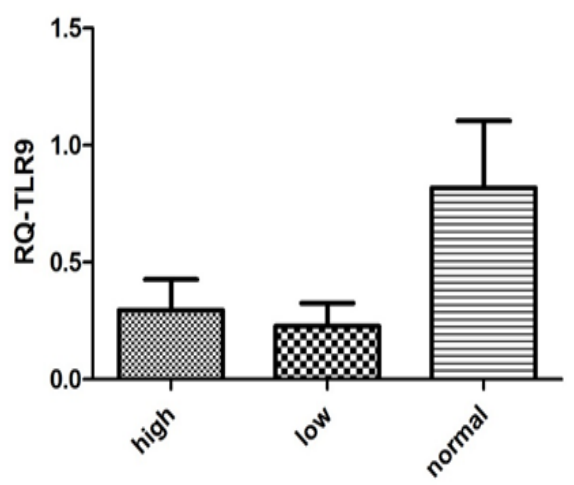

Figure 3. The Significant Down-Regulation of TLR9 mRNA in the High-Grade Dysplasia and Low-Grade Dysplasia versus the Normal Group $(P$-value $=0.002)$

control groups, diminished expression level of TLR9 was obtained in aAP in contrast to HP cases and normal individuals $(P$-value $=0.0008)$ (Figure 2$)$. Consequently, TLR9 $m R N A$ expression was evaluated in both high grade dysplasia (HGD) and low-grade dysplasia (LGD) in contrast to the control group, where lower expression levels were achieved for HGD and LGD $(P$-value $=0.002)$ (Figure 3). Finally, the level of TLR9 expression was lower in TP compared with TVP $(\mathrm{RQ}<0 / 05)$. This indicated significant differentiation between these polyp types $(P$-value $=0.004)($ Figure 4$)$.

\section{Discussion}

Investigation of the main molecular and immunological events in different types of polyp is important (Baxevanis et al., 2013) especially regarding early diagnosis of CRC and improving the management of patients. In the current study, it was found that TLR-9 mRNA expression was reduced in AP and HP cases compared with the normal individuals; hence, it was suggested that reduction of TLR-9 may play an important role in CRC initiation. Also, these data may support the development of TLR-9 agonists (synthetic oligodeoxynucleotides [ODNs]) for cancer treatment. However, the role of TLR9 in CRC pathology has not been fully elucidated up to now. Interestingly, we observed lower expression levels of TLR9 in HGD and

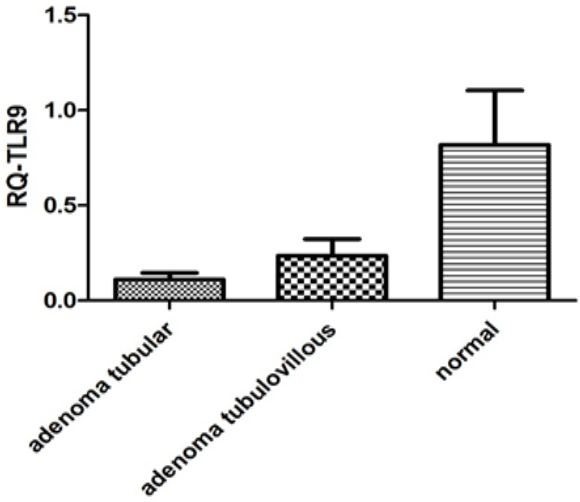

Figure 4. The Lower Expression of TLR9 mRNA in Tubular Adenoma Type Compared with the Normal Group and Tubulovillous Adenoma $(P$-value $=0.004)$.

$L G D$ compared with normal participants. Also, we found various levels of TLR9 $m R N A$ in different polyp types such as TP and TVP types. Our results were in contrast with Yesudhas et al., (2014) indicating that TLR7, 8, 9, and 10 mRNA expression increased in tissues of CRC patients. Indeed, they worked on CRC cases while the present study focused on different polyp cases; hence these differences may arise from differences between polyp and tumor tissues. On the other hand, Sandholm et al., (2014) demonstrated the beneficial role of TLR 9 on breast cancer treatment (Sandholm and Selander, 2014). Also, Shahriari et al., (2017) hypothesized that aberrant surface expression of TLR9 on tumor cells may promote tumor growth and invasion. They highlighted a dual contradictory role for CpG-ODNs, as an adjuvant agent in cancer therapy. Hasan et al., (2007) as well as Pacini et al., (2015) reported that downregulation of TLRO may be a crucial step in the carcinogenic events of human papillomavirus associated with cervical cancer. Furthermore, some researchers declared that oncogenic viruses such as papillomavirus 16, Epstein-Barr virus, and hepatitis B virus may have an important role in downregulating TLR9 expression (Fathallah et al., 2010; van Gent et al., 2011; Vincent et al. 2011). Finally, Jouhi et al., (2015) findings revealed diminishing expression levels of TLR 9 during Merkel cell carcinoma.

In conclusion, we observed decreasing TLR 9 expression in polyp cases of patients compared to normal samples. Also, lower expression level of TLR9 in HGD and $L G D$ compared to normal participants was achieved and different expression levels were obtained between TP and TVP. According to these results, it can be concluded that the reduction of TLR 9 expression may play a significant role in the progression of polyps to CRC and malignancy. Indeed, cancer cells may be able to escape the immune system due to TLR 9 reduction. Finally, TLR 9 modulation may have an impact on the development of novel therapeutic strategies.

\section{References}

Ansari R, Mahdavinia M, Sadjadi A, Nouraie M, et al (2006). Incidence and age distribution of colorectal cancer in Iran: results of a population-based cancer registry. Cancer Lett, 
240, 143-7.

Baxevanis CN, Papamichail M, Perez SA (2013). Immune classification of colorectal cancer patients: impressive but how complete?. Expert Opin Biol Ther, 13, 517-26.

Caixia G, Tiankui Q, Zhang B, et al (2018). TLR9 signaling activation at different stages in colorectal cancer and NF-kappaB expression. Onco Targets Ther, 11, 5963-5971.

Chen K, Huang J, Gong W, et al (2007). Toll-like receptors in inflammation, infection and cancer. Int Immunopharmacol, 7, 1271-85.

Fathallah I, Parroche P, Gruffat H, et al (2010). EBV latent membrane protein 1 is a negative regulator of TLR9. J Immunol, 185, 6439-47.

Haghdoost AA, Chamani G, Zarei MR, et al (2011). Low incidence of colorectal cancer in Kerman province, Iran. Iran J Cancer Prev, 4, 33-7.

Hale VL, Chen J, Johnson S, et al (2017). Shifts in the fecal microbiota associated with adenomatous polyps. Cancer Epidemiol Biomarkers Prev, 26, 85-94.

Hasan UA, Bates E, Takeshita F, et al (2007). TLR9 expression and function is abolished by the cervical cancer-associated human papillomavirus type 16. J Immunol, 178, 3186-97.

Inoue J, Gohda J, Akiyama T, Semba K (2007). NF-kappaB activation in development and progression of cancer. Cancer Sci, 98, 268-74.

Jouhi L, Koljonen V, Böhling T, Haglund C, Hagström J (2015). The expression of Toll-like receptors 2, 4, 5, 7 and 9 in Merkel cell carcinoma. Anticancer Res, 35, 1843-9.

Khatibi S, Nazemalhosseini Mojarad E, Forouzesh F, et al (2018). HIF-1 Alpha gene expression is not suitable biomarker for evaluating malignancy risk in colorectal polyps. WCRJ, 5, e1128.

Kim KM, Lee EJ, Ha S, et al (2011). Molecular features of colorectal hyperplastic polyps and sessile serrated adenoma/ polyps from Korea. Am J Surg Patho, 35, 1274-86.

Kolumam GA, Thomas S, Thompson LJ, et al (2005). Type I interferons act directly on CD8 T cells to allow clonal expansion and memory formation in response to viral infection. $J$ Exp Med, 202, 637-50.

Leslie A, Carey FA, Pratt NR, Steele RJ (2002). The colorectal adenoma-carcinoma sequence. Br J Surg, 89, 845- 60.

Liljegren A, Lindblom A, Rotstein S, et al (2003). Prevalence and incidence of hyperplastic polyps and adenomas in familial colorectal cancer: correlation between the two types of colon polyps. Gut, 52, 1140-7.

O'Connell B, Crockett SD (2017). The clinical impact of serrated colorectal polyps. Clin Epidemiol, 9, 113-25.

Pacini L, Savini C, Ghittoni R, et al (2015). Downregulation of toll-like receptor 9 expression by beta human papillomavirus 38 and implications for cell cycle control. J Virol, 89, 11396-405.

Peters BA, Dominianni Ch, Shapiro JA, et al (2016). The gut microbiota in conventional and serrated precursors of colorectal cancer. Microbiome, 4, 69.

Sandholm J, Selander KS (2014). Toll-like receptor 9 in breast cancer. Front Immunol, 5, 330-2.

Siegel RL, Miller KD, Jemal A (2016). Cancer statistics. $C A$ Cancer J Clin, 66, 7-30.

Shahriari Sh, Rezaeifard S, Moghimi HR, Khorramizadeh MR, Faghih Z (2017). Cell membrane and intracellular expression of toll-like receptor 9 (TLR9) in colorectal cancer and breast cancer cell-lines. Cancer Biomark, 1, 1-6.

van Gent M, Griffin BD, Berkhoff EG, et al (2011). EBV lytic-phase protein BGLF5 contributes to TLR9 downregulation during productive infection. $J$ Immunol, 186, 1694-702.

Vincent IE, Zannetti C, Lucifora J, et al (2011). Hepatitis B virus impairs TLR9 expression and function in plasmacytoid dendritic cells. PLoS One, 6, e26315.

Yesudhas D, Gosu V, Anwar MA, Choi S (2014). Multiple roles of toll-like receptor 4 in colorectal cancer. Front Immunol, 15,334 .

\section{(0) $(0)$}

This work is licensed under a Creative Commons AttributionNon Commercial 4.0 International License. 